Service social

\title{
Intervention et transmission intergénérationnelle
}

Services manquants, intervenants dépassés : l'intervention en

protection de la jeunesse et la transmission

intergénérationnelle de la maltraitance

\section{Geneviève Pagé et Jacques Moreau}

Volume 53, numéro 1, 2007

URI : https://id.erudit.org/iderudit/017988ar

DOI : https://doi.org/10.7202/017988ar

Aller au sommaire du numéro

\section{Éditeur(s)}

École de service social de l'Université Laval

ISSN

1708-1734 (numérique)

Découvrir la revue

Citer cet article

Pagé, G. \& Moreau, J. (2007). Intervention et transmission intergénérationnelle services manquants, intervenants dépassés : l'intervention en protection de la jeunesse et la transmission intergénérationnelle de la maltraitance. Service social, 53(1), 61-73. https://doi.org/10.7202/017988ar
Résumé de l'article

Les antécédents de maltraitance chez un des parents sont un facteur de risque fortement associé à la présence contemporaine de maltraitance, en particulier chez la clientèle suivie par la protection de la jeunesse (PJ). Le manque de connaissance sur la qualité, la quantité et l'efficacité de l'intervention en PJ faite auprès des familles maltraitantes sur plus d'une génération est questionnant. Cette étude a pour but l'analyse de la perception des intervenants en $\mathrm{PJ}$ face à cette question. Ces derniers manifestent une impuissance certaine puisqu'ils doivent entreprendre auprès de ces parents une intervention intensive, stable et à long terme, dans un contexte où ils sont surchargés de dossiers, où la continuité des services est bafouée et où la relation de confiance est difficile à établir. Une réflexion est amorcée sur la possibilité de briser le cycle de la transmission intergénérationnelle, compte tenu du mandat législatif de l'intervention en PJ. 


\title{
Intervention et transmission intergénérationnelle
}

\author{
Services manquants, intervenants dépassés : l'intervention en \\ protection de la jeunesse \\ et la transmission intergénérationnelle \\ de la maltraitance
}

Geneviève PAGÉ

Doctorat

École de service social

Université de Montréal

Jacques MOREAU

Professeur agrégé, Psychologie

École de service social, Université de Montréal

Les antécédents de maltraitance chez un des parents sont un facteur de risque fortement associé à la présence contemporaine de maltraitance, en particulier chez la clientèle suivie par la protection de la jeunesse (PJ). Le manque de connaissance sur la qualité, la quantité et l'efficacité de l'intervention en $\mathrm{PJ}$ faite auprès des familles maltraitantes sur plus d'une génération est questionnant. Cette étude a pour but l'analyse de la perception des intervenants en PJ face à cette question. Ces derniers manifestent une impuissance certaine puisqu'ils doivent entreprendre auprès de ces parents une intervention intensive, stable et à long terme, dans un contexte où ils sont surchargés de dossiers, où la continuité des services est bafouée et où la relation de confiance est difficile à établir. Une réflexion est amorcée sur la possibilité de briser le cycle de la transmission intergénérationnelle, compte tenu du mandat législatif de l'intervention en PJ.

Mots clés : transmission intergénérationnelle, intervention, maltraitance, protection de la jeunesse, perception des intervenants.

A parent's history of maltreatment is a highly associated risk factor to the presence of actual maltreatment, particularly within the child protective services (CPS) clientele. Considering the lack of knowledge in terms of CPS' quality, quantity, and effectiveness with intergenerational maltreating families, this study aims to analyse CPS workers' perceptions about this question. They demonstrate obvious helplessness considering intervention needs to be intensive, stable, and long-term with these parents, but within a context where practitioners are overloaded, where there is no continuity of services, and where a relationship based on trust is hard to establish. A reflection on the CPS' capacity to break the intergenerational transmission cycle, considering its legislative mandate, is undertaken.

Key words: intergenerational transmission, intervention, maltreatment, child protective services, perceptions of child protection services workers. 
Depuis le début des publications scientifiques sur le sujet de la maltraitance, les antécédents d'abus physique dans l'enfance du parent ont été identifiés comme étant un facteur fortement associé à la présence contemporaine de mauvais traitements de même nature (Kempe et al., 1962). De la même manière, les antécédents d'abus sexuel chez la mère constituent dans bien des cas un facteur de vulnérabilité, qui expose ses enfants au risque d'être victimes de ce même type de mauvais traitement (Black et al., 2001; Collin-Vézina et Cyr, 2003) ou de maltraitance chronique de façon plus générale (Éthier et al., 2004). Cette présence d'un vécu de maltraitance dans l'enfance du parent, jumelée à des gestes de maltraitance de la part de ce même parent envers ses propres enfants, est appelée la transmission intergénérationnelle de la maltraitance.

Des chercheurs ont tenté d'établir un taux de transmission, soit un pourcentage de cas où la maltraitance se répète dans la génération suivante. Les résultats varient énormément : dans certains cas, le taux est inférieur à $10 \%$, alors qu'il atteint jusqu'à $100 \%$ dans d'autres cas (Haapasalo et Aaltonen, 1999; Oliver, 1993). Buchanan (1998) de même que Haapasalo et Aaltonen (1999) et Oliver (1993), constatent que la prévalence de la transmission intergénérationnelle de la maltraitance est plus élevée dans les études rétrospectives que prospectives. De fait, il semble être établi un consensus selon lequel environ le tiers des enfants victimes d'abus (abused children) deviendront un jour des parents maltraitants (Belsky, 1993; Kaufman et Zigler, 1987; Langeland et Dijkstra, 1995; Oliver, 1993). De plus, il semble qu'un autre tiers des victimes demeurent vulnérables aux stress psychosociaux, donc à risque d'adopter des conduites parentales inadéquates (Oliver, 1993).

Dans le cas des taux rétrospectifs obtenus auprès des populations cliniques, telles que la clientèle suivie en protection de la jeunesse, ceux-ci se révèlent beaucoup plus élevés que les taux prospectifs (Haapasalo et Aaltonen, 1999). Les études répertoriées par Oliver (1993) obtiennent des taux variant de 75 à $100 \%$, alors que Buchanan (1998) note des taux de près de $100 \%$, principalement dans les études faites auprès des parents ayant été victimes de mauvais traitements sévères. Bref, les données rétrospectives permettent de croire qu'un nombre très élevé de parents maltraitants, particulièrement ceux connus par les autorités en protection de l'enfance, auraient des antécédents de maltraitance. II faut toutefois considérer ces chiffres avec prudence, puisque plusieurs failles méthodologiques ont été relevées pour tenter d'expliquer la grande variance des taux (Ertem et al., 2000). Cette variance signifie surtout que la transmission intergénérationnelle de la maltraitance ne se résume pas uniquement à la croyance populaire voulant que la violence engendre la violence (violence begets violence). En effet, l'histoire de maltraitance dans l'enfance du parent n'est ni un facteur nécessaire, ni un facteur suffisant, pour expliquer ces comportements de maltraitance envers ses propres enfants (Widom, 1989). Mais il n'en demeure pas moins qu'il s'agit là d'une réalité inquiétante, pour laquelle l'intervention en protection devient un outil important à considérer.

Très peu d'études ont comme objet de recherche l'intervention auprès des parents maltraitants qui ont eux-mêmes subi de mauvais traitements dans leur enfance. En 
effet, on a recensé seulement l'étude de Rocklin et Lavett (1987), dont l'objectif principal était de décrire le processus thérapeutique et les particularités d'une dizaine de parents qui ont subi de mauvais traitements physiques dans leur enfance, sans pour autant répéter ce genre de comportements auprès de leurs propres enfants. Autrement, quelques rares auteurs font l'analyse de certaines pratiques d'intervention spécifiques aux familles reproductrices de la maltraitance (Doré, 1994; Emery et LaumannBillings, 1998; Karson, 2001). Par exemple, certains programmes éducatifs ont été développés afin de tenter de prévenir la récurrence de la maltraitance (Emery et Laumann-Billings, 1998). Ces interventions ciblent les parents maltraitants ou à risque de l'être, tels que des parents ayant eux-mêmes subi de la maltraitance dans leur enfance (Wilkes, 2002). Cependant, il semble que plus la maltraitance est de nature sévère et chronique, moins ces programmes d'intervention s'avèrent efficaces (Emery et Laumann-Billings, 1998) et, également, moins le lien entre la chronicité et la transmission intergénérationnelle de la maltraitance tend à être démontré (Boulet et al., 2004; Éthier et al., 2004). De son côté, Karson (2001) décrit la dynamique de la transmission intergénérationnelle de la maltraitance selon une approche systémique et présente des principes d'intervention, tels que la nécessité d'agir avec le système familial plutôt que sur le système familial, afin de modifier les patrons de comportements dysfonctionnels et rigides comme la maltraitance. Ces interventions éducatives et systémiques ne prennent toutefois pas en considération certains aspects importants de la réalité des parents maltraitants qui ont eux-mêmes eu une enfance marquée d'abus, tels que la souffrance psychologique liée à leur trajectoire de vie.

Selon Doré (1994) et Rocklin et Lavett (1987), le fait d'entreprendre une démarche thérapeutique est la meilleure voie à prendre afin d'éliminer la transmission de la maltraitance. L'intervention peut amener une prise de conscience des parents qui ont été marqués par leur expérience de maltraitance, afin de modifier leurs perceptions et leurs comportements, et d'aider ainsi à briser le cycle de la transmission.

Lorsqu'il s'agit de parents maltraitants ayant eux-mêmes des antécédents de ce genre, « toute démarche qui vise à un changement de cap ne peut se faire que graduellement et s'avère lourde d'exigences, puisqu'un déséquilibre se trouve provoqué chez le sujet qui tente d'arrêter la reproduction de comportements problématiques dans sa vie » (Doré, 1994, p. 323). Ce long processus thérapeutique peut se diviser en cinq étapes: 1) prendre conscience de la répétition et des mécanismes sous-jacents; 2) s'autoriser à se souvenir d'un passé douloureux; 3) s'autoriser à ressentir les émotions que ce passé fait ressurgir; 4) se donner le droit d'être différent du système familial; et 5) faire le deuil de l'amour et d'un passé idéalisés (Doré, 1994). La relation entre le thérapeute et son client est dans ce cas-ci d'une extrême importance. En effet, le client a besoin de soutien émotionnel et cognitif intense, ce qui demande au thérapeute d'avoir un rôle plus actif durant la thérapie (Rocklin et Lavett, 1987).

Parmi les parents qui perpétuent le cycle de la maltraitance dans la génération suivante, très peu se présentent en thérapie, entre autres, parce qu'ils se retrouvent souvent parmi la classe sociale la plus défavorisée. Ils font plutôt face à l'intervention de la Direction de la protection de la jeunesse (DPJ), lorsque leur situation est signalée, et, 
pourtant, ce type d'intervention semble très peu documenté. Lorsqu'on réfère aux interventions en protection de l'enfance de manière plus générale, une recension critique sur leur efficacité (Dufour et Chamberland, 2003) conclut que ces dernières ont été très peu évaluées, faisant en sorte que des connaissances plus poussées manquent dans ce domaine. De plus, peu d'évaluations mesurent le maintien dans le temps des améliorations dues à l'intervention lorsque celle-ci est terminée (Dufour et Chamberland, 2003). D'autre part, bien que certaines interventions évaluées soient considérées comme efficaces en ce qui a trait à la diminution des comportements de maltraitance, cette baisse observée repose principalement sur des différences statistiques, ce qui ne représente pas nécessairement une différence significative sur le plan clinique (Éthier et Lacharité, 2003). Bref, il est difficile de savoir si l'intervention en protection de la jeunesse auprès des familles permet d'éviter la récurrence des comportements de maltraitance, et ceci est encore plus difficile à établir sur plus d'une génération.

Compte tenu du manque de connaissance en ce qui a trait à la qualité, la quantité et l'efficacité de l'intervention en protection de la jeunesse faite auprès des familles qui reproduisent la maltraitance de génération en génération, il serait intéressant de savoir ce qu'en pensent les intervenants en protection de la jeunesse eux-mêmes. Le regard de ces professionnels, qui œuvrent directement auprès de la clientèle, a été peu pris en considération dans les recherches. La présente étude a pour but l'analyse des perceptions des intervenants en protection de la jeunesse face à leurs interventions auprès des familles aux prises avec une dynamique de transmission intergénérationnelle de la maltraitance. De façon plus précise, il est question de décrire et de mieux comprendre leur manière particulière d'intervenir auprès de ces familles, leurs perceptions de ce type d'intervention dans un contexte idéal et les obstacles à l'intervention auprès des familles reproductrices de maltraitance dans le contexte actuel.

\section{MÉTHODOLOGIE}

Afin de répondre à l'objectif de la présente recherche, 11 intervenants d'un centre jeunesse $(\mathrm{CJ})$ participent à une entrevue semi-structurée, d'une durée moyenne de 40 minutes. De l'information concernant l'étude est préalablement transmise aux chefs d'équipe des différents services : évaluation/orientation (É/O), application des mesures (AM) et révision (R). Après avoir pris connaissance de l'objectif de la recherche, ces derniers ont sollicité la participation des membres de leur équipe, soit sur une base individuelle, soit en faisant un appel à tous. La participation se fait sur une base volontaire. Tous les intervenants qui se sont montrés intéressés à participer à la recherche font partie de l'échantillon. Le tableau 1 présente les différentes caractéristiques de ce dernier. Ces informations sont recueillies grâce à un questionnaire autoadministré. 
TABleau 1. CARActéRISTIQUes des PARTICIPANTS

\begin{tabular}{lccccccccccc}
\hline Intervenant & 1 & 2 & 3 & 4 & 5 & 6 & 7 & 8 & 9 & 10 & 11 \\
\hline Sexe & $\mathrm{F}$ & $\mathrm{M}$ & $\mathrm{M}$ & $\mathrm{F}$ & $\mathrm{F}$ & $\mathrm{F}$ & $\mathrm{F}$ & $\mathrm{F}$ & $\mathrm{F}$ & $\mathrm{F}$ & $\mathrm{F}$ \\
Âge & $40-49$ & $40-49$ & $50-59$ & $40-49$ & $40-49$ & $60-69$ & $40-49$ & $40-49$ & $30-39$ & $50-59$ & $40-49$ \\
Poste & É/O & É/O & É/O & É/O & É/O & $\mathrm{R}$ & $\mathrm{R}$ & $\mathrm{AM}$ & $\mathrm{AM}$ & $\mathrm{AM}$ & AM \\
Années CJ & 15 & 19 & 23 & 16 & 15 & 24 & 27 & 23 & 17 & 27 & 17 \\
Formation & T.S. & Crim. & T.S. & T.S. & T.S. & Psy. & T.S. & Éduc. & T.S. & T.E.S. & T.S. \\
\hline
\end{tabular}

Ainsi, la majorité des intervenants sont des femmes entre 40 et 49 ans. Ils proviennent principalement des services évaluation/orientation et de l'application des mesures, mais aussi de la révision. Ils ont entre 15 et 27 années d'expérience en $\mathrm{CJ}$, pour une moyenne de 20,27 ans. Concernant leur parcours professionnel, six des onze intervenants ont travaillé uniquement en $\mathrm{CJ}$. Les cinq autres ont occupé divers emplois avant les $\mathrm{CJ}$, soit en enseignement, en psychopédagogie, dans le domaine communautaire ou en bureau privé. Finalement, la majorité ont une formation en travail social.

La grille d'entrevue se compose de 10 questions ouvertes et d'une question fermée, regroupées sous quatre thèmes. L'entrevue vise à recueillir les réponses spontanées des intervenants. Les thèmes abordés avec les intervenants sont regroupés selon leur lien avec la conception théorique de la transmission intergénérationnelle de la maltraitance, ou avec la pratique auprès de cette clientèle particulière. La conception théorique des intervenants concernant la transmission intergénérationnelle de la maltraitance n'est pas abordée ici, car elle constitue le sujet d'un autre article. Sur le plan de la pratique, les thèmes abordés sont: l'intervention actuelle, l'intervention dans un contexte idéal, et les facteurs facilitant ou limitant la portée de l'intervention dans le contexte actuel. Cette grille a été élaborée par la première auteure pour les besoins de l'étude. La validité et la clarté des questions de l'entrevue ont été soumises à une procédure d'approbation par des experts, dont le directeur de recherche.

Les verbatim d'entrevues sont soumis à une analyse de contenu constitué. Le matériel est ainsi codifié en «noyaux de sens », puis catégorisé selon les questions de la grille d'entrevue. Cette méthode permet de découvrir la signification du discours des intervenants rencontrés en lien avec les thèmes choisis (Mayer et Deslauriers, 2000). 


\section{RÉSULTATS}

\section{L'INTERVENTION DANS LES CAS DE TRANSMISSION INTERGÉNÉRATIONNELLE DE LA MALTRAITANCE}

Huit des onze intervenants s'entendent sur le fait que les antécédents de maltraitance du parent sont un fait important à noter pour l'intervention auprès de la famille. L'histoire du parent est d'ailleurs l'une des premières thématiques explorée à la suite des détails de la situation de compromission. Le passé permet de mieux expliquer ce qui se passe dans le présent et permet de spéculer sur ce qui risque d'arriver dans l'avenir.

Pour la majorité des intervenants, une part importante de l'intervention consiste en l'évaluation de la capacité du parent à reconnaître qu'il a un passé d'abus et que cette expérience a un impact sur sa façon d'être parent dans le présent. L'intervenant doit amener le parent à cheminer vers cette prise de conscience : « [...] vraiment, moi, je trouve que dire les choses là, par rapport à [...] l'impact que ça a eu sur l'enfant, que ça a sur son enfant, faut le dire aussi, pis faut le dire clairement, pis faut pas, faut pas ménager. Parce que ça a un impact qui est clair, qui est direct, qui est certain. » (Int. 4)

Une autre particularité de l'intervention auprès des parents maltraitants ayant des antécédents de maltraitance est le fait de devoir amener le parent à reprendre contact avec la souffrance qu'il a vécue lors de son enfance. Ce parent doit comprendre que son vécu doit être surmonté et que le bien-être de son enfant en dépend directement. Cependant, l'intervenant se doit d'être prudent afin de ne pas aborder avec le parent certaines souffrances que celui-ci ne sera pas capable de gérer. II ne peut pas se prétendre psychothérapeute s'il ne possède pas ces habiletés : « [...] il faut faire attention quand même de ne pas rouvrir d'anciennes blessures sans être sûr qu'on est capable d'aider aussi, qu'on est capable d'apaiser leurs souffrances là, parce que, on pourrait faire plus de mal que de bien. » (Int. 2)

Suite à l'évaluation de la reconnaissance et à la reprise de contact avec la souffrance du parent, l'intervenant doit faire une lecture juste et rapide des capacités parentales. II s'agit en fait de voir jusqu'à quel point le parent est fonctionnel malgré son vécu douloureux. II est possible que l'intervenant soit appelé à aider le parent à développer de nouvelles habiletés s'il y a des lacunes à ce niveau : «On devrait apprendre à être parent. Ce n'est pas nécessairement un phénomène inné. Parce que [...] je veux dire, il y a des choses à apprendre au parent. L'apprentissage du développement d'un enfant aussi. » (Int. 6)

Selon certains intervenants, il est important de vérifier l'ouverture du parent à recevoir de l'aide. Afin d'évaluer sa volonté de changer, l'intervenant peut se fier au fait que le parent a entrepris une ou plusieurs démarches d'aide dans le passé. S'il n'a pas eu recours à des services, le parent peut être dirigé vers une démarche personnelle afin de régler son passé non résolu. C'est à partir de l'histoire du parent qu'il est possible 
pour l'intervenant de savoir s'il peut donner une chance au parent ou non : « Quand on regarde l'histoire, pis on regarde le vécu, pis on regarde l'aide antérieure, pis bon, ça, ça nous donne des indices qui nous permettent de croire ou pas au parent, et de se permettre de patienter ou pas auprès de l'enfant. » (Int. 2)

L'intervention doit être directe et contraignante afin d'exercer une pression sur le parent à changer rapidement. Toutefois, les praticiens disent qu'ils doivent être patients dans leur travail avec cette clientèle particulière, parce que l'intervention est lourde et à long terme. Le parent est très souffrant face à son passé et a beaucoup à réapprendre. Neuf intervenants sur onze manifestent une impuissance certaine, puisqu'ils ne savent pas concrètement comment défaire le cycle de la transmission de la maltraitance : « Mais, c'est peut-être ce qui me frappe le plus, comment c'est difficile à défaire. Comment c'est ben, ben difficile de renverser, de renverser la vapeur. » (Int. 9)

Étant donné la difficulté d'intervenir auprès des parents, plus de la moitié des praticiens disent qu'ils orientent leur travail dans le meilleur intérêt de l'enfant. Leur crainte est que, si trop de chances sont laissées au parent, l'enfant en subisse les conséquences : «[...] je dirais que, au départ, le pronostic est assez sombre parce que les capacités de changement sont relativement faibles, alors... on va plus travailler sur l'enfant, si l'enfant n'est pas trop détérioré. » (Int. 6)

Ainsi, les intervenants sont moins tolérants face à ce parent, comparativement à un parent sans passé de maltraitance, et ils tiennent à intervenir rapidement. Ils considèrent important de ne pas créer de l'espoir chez le parent pour rien, c'est-à-dire, s'ils ne croient pas que ce dernier puisse changer. Afin de protéger l'enfant, le placement en famille d'accueil ou le projet de vie permanent ${ }^{1}$ sont souvent vus comme les seules solutions aux yeux des intervenants: "Ça brise des vies, parce que tu renoues l'espoir tout le temps, [...] mais l'espoir, tu sais! C'est pas possible. Au lieu d'avoir statué dès le point de départ, pis de faire un projet [de vie permanent] pour cet enfantlà. » (Int. 7)

\section{L'INTERVENTION DANS UN CONTEXTE IDÉAL}

Interrogés sur les interventions à préconiser dans un contexte idéal, certains intervenants souhaiteraient agir de façon précoce et qu'il y ait davantage de prévention et de dépistage. Si cela était possible, il faudrait intervenir intensivement avec la clientèle où la maltraitance est transmise à travers plus d'une génération. Pour la moitié des praticiens interrogés, il faudrait pouvoir travailler en même temps avec plus d'une génération à la fois, c'est-à-dire, avec l'enfant, le parent et le grand-parent.

1. Un «projet de vie » est une situation dans laquelle l'enfant est placé de façon stable et permanente. Cette situation comporte deux dimensions: une dimension physique (un milieu de vie, un lieu d'appartenance) et une dimension dynamique (une personne significative avec qui l'enfant vit et peut développer un lien d'attachement) (Paquette, 2004, p. 12). 
La moitié des intervenants perçoivent également que l'idéal serait d'obtenir plus facilement la reconnaissance et la collaboration du parent. Enfin, l'intervention de groupe serait, selon certains, un bon moyen pour arrêter le cycle de transmission, en brisant l'isolement et en favorisant l'entraide. " C'est sûr qu'avant, quand on travaillait avec le programme en abus sexuel, on avait des groupes de mères. Et, on avait cette approche-là de travailler par rapport à leur enfance, pis regarder au niveau de la transmission intergénérationnelle. Et les parents embarquaient beaucoup là-dedans. » (Int. 10)

\section{LES LIMITES DE L'INTERVENTION}

Sur ce qui facilite ou limite leurs interventions dans le contexte actuel de travail, seule la capacité à faire son travail de façon autonome est identifiée par quelques intervenants comme aspect positif. II semble cependant que les intervenants en ont davantage à dire sur les limites de l'intervention auprès des familles aux prises avec la transmission intergénérationnelle de la maltraitance.

Tout d'abord, la majorité des intervenants reprochent à l'employeur de leur donner trop de dossiers à traiter. Ils manquent alors de temps pour intervenir de façon satisfaisante auprès des familles. Ceci peut entraîner plusieurs conséquences, telles que la prise de mauvaises décisions, le fait que certaines actions sont entreprises trop tard, de même que la difficulté d'établir une relation de confiance : « Mais aujourd'hui là, quand même que les services sociaux voudraient là, on a rien! On a rien! On a quoi ? Une heure ou deux d'intervention par 15 jours, trois semaines. C'est pas suffisant ça. C'est pas suffisant. Pas du tout. » (Int. 7)

Les intervenants considèrent qu'il y a un manque de services adéquats et accessibles pour les familles. Ils constatent aussi une discontinuité dans l'intervention, causée par un manque de concertation entre les divers professionnels impliqués auprès de ces familles: « Une garderie, ça prend des mois et des années d'attente, un service en psychologie avec un psychologue, un pédopsychiatre, ça peut prendre des mois et des mois d'attente. » (Int. 2)

Plus de la moitié des intervenants se plaignent d'un manque de formation et de lacunes en termes de compétences des praticiens. C'est qu'ils font face à des situations multiproblématiques dont ils n'arrivent pas à travailler tous les aspects en même temps. Ils dénoncent également un manque d'outils adéquats pour intervenir auprès de ces familles et un manque d'accessibilité au soutien clinique.

Une autre difficulté identifiée par les intervenants est que, même si certains enfants sont placés, il n'est pas possible de les « sauver » de la transmission intergénérationnelle, parce qu'ils sont souvent retournés dans leur famille après un certain temps. II n'y a donc pas de coupure définitive avec le modèle familial de maltraitance : «Parce que même quand on coupe les contacts, des fois, tu sais, ces enfants-là ont tellement mal à leurs parents, qu'ils en ont besoin, même si les parents n'ont pas d'allure. Pis, qui me dit qu'à 18 ans, que le jeune n'ira pas vivre chez ces parents-là ? » (Int. 8) 
Finalement, quelques intervenants étiquettent l'intervention de la DPJ comme étant une intervention de surface qui ne s'attaque pas au cœur de ce problème.

Donc, dans le fond on est appelé souvent à... à trouver une solution du moindre mal. Mais on ne règle pas nécessairement les bonnes affaires au bon moment. Des fois, on étire, on temporise, on travaille le symptôme, on rend les... on essaie d'aider suffisamment pour que les gens deviennent suffisamment fonctionnels, même si les problèmes sont loin d'être réglés, pis que les inquiétudes demeurent, ok. Mais, on essaie de gagner du temps. (Int. 2)

Certains vont même jusqu'à dénoncer le fait que les failles du système de protection permettent à ces familles de se maintenir dans une dynamique de transmission intergénérationnelle de la maltraitance.

Là, c'est nous qui devenons intergénérationnels [...] Parce que c'est quand même grave que des enfants DPJ fassent des enfants DPJ. (Int. 6)

Oui, on a une mission de protéger. Mais, en même temps, si on veut éliminer la récurrence, il faudrait travailler sur nos problèmes. (Int. 8)

\section{DISCUSSION}

Selon le discours des intervenants en protection de la jeunesse, la modification d'une dynamique familiale intergénérationnelle de maltraitance s'opère nécessairement par la reconnaissance de la transmission intergénérationnelle par le parent, et également par la prise de contact avec la souffrance que ce dernier a vécue lorsqu'il était enfant. Ce n'est que lorsque ces étapes préalables sont franchies que peut se faire l'acquisition de nouvelles habiletés parentales.

Les praticiens cernent correctement les besoins des familles maltraitantes sur plus d'une génération en termes d'intervention, comme il a été mentionné dans les écrits scientifiques et cliniques. En effet, ces gens nécessitent une intervention à long terme, qui respecte leur rythme et où le lien de confiance, bien que lent à établir, est primordial, afin de leur permettre de ressasser des souvenirs douloureux en toute sécurité (Doré, 1994; Rocklin et Lavett, 1987). Toutefois, la loi fait en sorte que l'intervention en protection doit être rapide dans le temps et que le parent doit démontrer sa capacité à se mobiliser très tôt, sans quoi il risque de voir ses enfants placés en famille d'accueil. Les intervenants ressentent donc beaucoup d'impuissance face à la transmission intergénérationnelle de la maltraitance, puisqu'ils sont face à un dilemme entre la difficulté du parent de se mobiliser et le besoin de protection de l'enfant.

Les intervenants rencontrés dressent un pronostic plutôt sombre quant à la possibilité des familles de se sortir du cycle de maltraitance. En fait, ils ne se sentent pas adéquatement outillés pour entreprendre l'intervention ardue dont ces parents ont besoin. Ils sont coincés par leur contexte actuel de travail, qui ne leur permet pas d'investir suffisamment de temps, ne leur fournit pas les services et l'expertise nécessaires, et 
ne leur permet pas d'intervenir en continuité auprès de ces familles. Cette perception semble fidèle à la réalité. En effet, alors que le nombre de dossiers d'enfants pris en charge par un intervenant en protection de la jeunesse devrait normalement être de 16 (norme établie par la Child Welfare League of America), la moyenne provinciale actuelle se situe plutôt autour de 23 dossiers par intervenant (ACJQ, 2005). Le fait que certains dossiers contiennent probablement des problématiques complexes et chroniques ne peut qu'alourdir davantage la tâche de ces intervenants débordés.

Les résultats obtenus dans la présente étude permettent de questionner l'efficacité de l'intervention de la DPJ dans les cas de transmission intergénérationnelle de la maltraitance. D'une part, la Loi sur la protection de la jeunesse (LPJ) stipule que « toute intervention auprès d'un enfant et de ses parents doit viser à mettre fin à la situation qui compromet la sécurité ou le développement de l'enfant et à éviter qu'elle ne se reproduise » (LPJ, art. 2.3). Bien que la dernière partie de cet article de loi réfère à la récurrence d'une même situation de maltraitance au sein d'une même génération, il est possible d'en élargir l'interprétation à la reproduction de la maltraitance d'une génération à l'autre. D'ailleurs, certains CJ prônent explicitement l'évitement du transfert intergénérationnel des problématiques avec lesquelles les familles sont aux prises dans leur philosophie d'intervention (Centre jeunesse de Montréal, 2002).

Cependant, les familles où la problématique de la maltraitance est reproduite de génération en génération représentent une clientèle dont les besoins à combler en termes de services sont importants. La réalité d'intervention des $\mathrm{CJ}$ ne permet pas une intervention intensive et à long terme, avec un intervenant entièrement disponible, puisque les intervenants sont débordés, les ressources sont en pénurie et la stabilité des intervenants et des services est difficile à obtenir. II semble donc que la faute ne doive pas tant être accordée aux intervenants qu'aux maigres ressources dont l'organisme dispose et à la façon dont celles-ci sont gérées.

À ce sujet, il est possible d'établir un lien entre la transmission intergénérationnelle et la chronicité de la maltraitance, ou, à tout le moins, de la négligence. En effet, selon Boulet, Éthier et Couture (2004), les événements traumatiques vécus par la mère dans son enfance, particulièrement s'ils demeurent non résolus à l'âge adulte, seraient associés à la persistance de comportements de négligence de cette dernière, et ce, malgré une intervention de plusieurs années en protection de la jeunesse. Ainsi, cette corrélation pourrait permettre d'expliquer la difficulté d'intervenir auprès des familles où la négligence, et probablement d'autres formes de mauvais traitements, se reproduit de génération en génération. De fait, le type, l'intensité et la quantité de l'intervention, de même que l'expérience de l'intervenant, ne seraient pas suffisants pour diminuer significativement les comportements de négligence de ces parents (Gelles, 2000, dans Boulet et al., 2004).

Un autre élément permet de comprendre pourquoi l'intervention de la DPJ réussit difficilement à freiner la transmission intergénérationnelle de la maltraitance. II importe de se remémorer les principes de la LPJ afin de bien saisir là où le pouvoir d'intervention de la DPJ débute et là où il se termine. La LPJ est une loi d'exception. En effet, elle ne 
s'applique pas à la population générale, mais bien à une clientèle en particulier, soit les enfants dont la sécurité et le développement sont compromis. Ainsi, «la protection d'un enfant consiste à apporter une réponse minimale à ses besoins fondamentaux, dans son meilleur intérêt, et dans le respect de ses droits » (Boucher et al., 1995, p. 4). L'intervention des $\mathrm{CJ}$, selon les dispositions de la LPJ, vise avant tout à corriger une situation de compromission (ibid.). Par conséquent, à partir du moment où les faits en cause, soit les comportements de maltraitance des parents, se résorbent, les intervenants en protection se retirent de la vie de cette famille et n'ont plus le pouvoir d'intervenir. Or, lorsqu'il est question de transmission intergénérationnelle de la maltraitance, la situation ne se limite pas uniquement à des comportements de maltraitance. II s'agit d'un contexte de vie familiale, ancré depuis plusieurs générations. Comme l'intervention en protection est axée sur l'extinction de comportements maltraitants, les conditions de vie et les traumas non résolus ne sont quasiment pas travaillés, alors qu'ils devraient être au centre du processus d'intervention. Tant qu'il en sera ainsi en protection de la jeunesse, la maltraitance demeurera un problème qui se répète, dans une très grande proportion, de génération en génération. II faut rapidement constater qu'une intervention minimale est nettement insuffisante dans le cas de ces familles, où les problématiques sont multiples, complexes et chroniques. Criante est la nécessité de développer davantage le travail intersectoriel et de développer des expertises dans ce domaine.

La présente étude comporte certaines limites. Dans un premier temps, la participation des intervenants se faisait sur une base volontaire. Ainsi, ceux qui ont bien voulu participer à l'étude étaient déjà intéressés par le sujet. II est donc probable que leurs perceptions ne pourraient être généralisées à l'ensemble des intervenants en protection de la jeunesse. Le constat est le même, compte tenu du fait que la majorité des intervenants étaient de sexe féminin, âgés entre 40 et 49 ans, et formés en travail social. II s'agit aussi d'intervenants expérimentés; par conséquent, ils n'avaient peut-être pas les mêmes perceptions du phénomène que les intervenants moins expérimentés. Enfin, comme il s'agit d'une analyse du discours des intervenants, il ne faut pas perdre de vue le fait qu'il pouvait exister un écart entre leurs perceptions et la réalité.

Davantage de recherches sont nécessaires afin de mieux connaître les interventions les plus efficaces pour briser le cycle de la transmission intergénérationnelle de la maltraitance. Si les familles maltraitantes à travers les générations résistent à l'intervention en protection de la jeunesse, alors les moyens à prendre afin de remédier à cette situation ne relèvent certainement pas uniquement de la DPJ. II importe de mieux comprendre les facteurs qui perpétuent le cycle, afin que la société adopte des politiques visant à offrir aux familles vulnérables de meilleures opportunités de vie. 


\section{RÉFÉRENCES BIBLIOGRAPHIQUES}

ASSOCIATION DES CENTRES JEUNESSE DU QuÉBEC (2005). Les voleurs d'enfance : des réponses à des questionnements soulevés par le documentaire, communiqué de presse, 4 octobre 2005. [http://www.acjq.qc.ca/].

BELSKY, J. (1993). «Etiology of child maltreatment: A developmental-ecological analysis », Psychological Bulletin, vol. 114, $\mathrm{n}^{\circ} 3$, p. 413-434.

Black, D.A., R.E. HeYman, et A.M. SMith SLEP (2001). « Risk factors for child sexual abuse », Aggression and Violent Behavior, vol. 6, p. 203-229.

Boucher, L., R. Crête, R. Dupont, D. Gauthier, M. Lacour, H. Ostiguy, et D. Poirier (1995). Concept de protection et interprétation des articles 38 et 38.1 de la Loi sur la protection de la jeunesse, Québec, Association des Centres jeunesse du Québec, 32 p.

Boulet, M.-C., L.S. ÉTHIER, et G. COUTURE (2004). « Événements de vie et traumatismes chez les mères négligentes chroniques », Santé mentale au Québec, vol. XXIX, n 1, p. 221-242.

BUCHANAN, A. (1998). «Intergenerational child maltreatment » dans Y. DANIELI (dir.), International Handbook of Multigenerational Legacies of Trauma, New York, Plenum Press, p. 535552.

CentRe JeUnesse de MontRÉAl (2002). Formation sur l'application de la Loi sur la protection de la jeunesse. Secteur psychosocial, collection Formation, Montréal, Centre jeunesse de Montréal, $48 \mathrm{p}$.

COLLIN-VÉZINA, D., et M. CYR (2003). « La transmission de la violence sexuelle : description du phénomène et pistes de compréhension », Child Abuse et Neglect, vol. 27, p. 489-507.

DORÉ, L. (1994). « La répétition intergénérationnelle ou la résistance au changement de cap », P.R.I.S.M.E., vol. 4, $\mathrm{n}^{\mathrm{os}} 2-3$, p. 312-328.

Dufour, S., et C. ChAmBERLAND (2003). L'efficacité des interventions en protection de l'enfance. Recension des écrits, Montréal, Centre d'excellence pour la protection et le bienêtre des enfants, $47 \mathrm{p}$.

EMERY, R.E., et L. LAUMANN-BILLINGS (1998). « An overview of the nature, causes, and consequences of abusive family relationships », American Psychologist, vol. 53, p. 121-135.

ERTEM, I.O., J.M. Leventhal, et S. Dobbs (2000). « Intergenerational continuity of child physical abuse: How good is the evidence? », The Lancet, 356, p. 814-819.

ÉtHIER, L.S., G. CoutURE, et C. LACHARITÉ (2004). « Risk factors associated with the chronicity of high potential for child abuse and neglect », Journal of Family Violence, vol. $19, \mathrm{n}^{\circ} 1$, p. 13-24.

HaApasalo, J., et T. Aaltonen (1999). « Mothers' abusive chilhood predicts child abuse », Child Abuse Review, vol. 8, p. 231-250.

KARSON, M. (2001). «Part IV: Intervention patterns », in Patterns of Child Abuse: How Dysfunctional Transactions Are Replicated in Individuals, Families, and the Child Welfare System, New York, Haworth Maltreatment and Trauma Press, p. 177-234.

KAUFMAN, J., et E. ZigleR (1987). « Do abused children become abusive parents? », American Journal of Orthopsychiatry, vol. 57, $\mathrm{n}^{\circ} 2$, p. 186-192. 
Kempe, C.H., F.N. Silverman, B.F. Steele, W. Droegemueller, et H.K. Silver (1962). « The battered-child syndrome », JAMA: Journal of the American Medecine Association, vol. 181, $\mathrm{n}^{\circ} 1$, p. 17-24.

LANGELAND, W., et S. DIJKSTRA (1995). «Breaking the intergenerational transmission of child abuse: Beyond the mother-child relationship », Child Abuse Review, vol. 4, p. 4-13.

MAYER, R., et J.-P. DESLAURIERS (2000). «Quelques éléments d'analyse qualitative : l'analyse de contenu, l'analyse ancrée, l'induction analytique et le récit de vie », dans R. MAYER et al., Méthodes de recherche en intervention sociale, Boucherville, Gaëtan Morin, p. 159-189.

OLIVER, J.E. (1993). « Intergenerational transmission of child abuse: Rates, research, and clinical implications », American Journal of Psychiatry, vol. 150, $n^{\circ}$ 9), p. 1315-1324.

PAQuetTE, F. (2004). À chaque enfant son projet de vie permanent: un programme d'intervention, Montréal, Centre jeunesse de Montréal - Institut universitaire, $144 \mathrm{p}$.

RockLIN, R., et D.K. LAVETT (1987). « Those who broke the cycle: Therapy with nonabusive adults who were physically abused as children », Psychotherapy, vol. 24, n 4, p. 769-778.

WIDOM, C.S. (1989). « Does violence beget violence? A critical examination of the literature », Psychological Bulletin, vol. 106, $\mathrm{n}^{\circ}$ 1, p. 3-28. 